\title{
Development of the physics of microwaves, and its unification with infrared and optical science
}

Charles H. Townes

cht@ssl.berkeley.edu
University of California Berkeley, California, USA

Radio waves and light for a long time seemed quite separated, one primarily a field of engineering, and the other more a field of science. Now they have come together, with engineering and basic science strongly involved in both. This change originated largely through the development of microwave physics, which led to laser oscillators and amplifiers. The whole electromagnetic spectrum, from radio waves to the ultraviolet and beyond, now looks like a continuous field from both a scientific and engineering point of view. The development of microwave physics, an essential part of this story, will be discussed here with its impact on masers, lasers, and optics. [DOI: 10.2971/jeos.2010.10043s]

Keywords: microwaves, infrared

I took my first job in 1939, just before World War II, at the Bell Telephone Laboratories expecting to do fundamental physics. I did do physics initially, but after a little more than a year, Bell Labs boss assigned me to work on radar. I didnt really want to do such engineering, but everyone felt we must try to help win World War II, and I was assigned the job of developing a radar of about 1 centimeter wavelength, a shorter wavelength than any then available and hence it could be more directional. After some time developing such a system, we recognized that our wavelength of $1.25 \mathrm{~cm}$ was absorbed by water vapor in the atmosphere, so it wouldnt work as a radar. Oh dear!

I spent some time studying this water vapor absorption, and as a result recognized that one could do very high resolution and useful spectroscopy on molecules using microwaves. After the war, at my urging Bell Labs allowed me to do research on such spectroscopy, and I initiated it with measurements on the spectrum of ammonia. The high resolution results allowed very precise measurement of the structure of molecules, the mass ratios of nuclei in them, and also the spin and nonsphericity (quadrupole moments) of these nuclei. It was exciting, and the work resulted in my being offered a job as professor in the physics department at Columbia University, where I continued this work.

After some years of exciting research with waves in the centimeter region, I wanted to get to shorter wavelengths to extend the research down to wavelengths less than one millimeter, and on into the infrared. My students and I worked on several ideas for producing oscillators that would work at shorter waves than anyone could then produce and provide very high spectral resolution, but our efforts were unsuccessful. Finally, I suddenly had the right idea use molecules or atoms, which of course everyone knew oscillate at high frequencies. But the new idea was to put them in a state contrary to the usual thermo-dynamic equilibrium which can give us only an intensity limited by the temperature, and not amplify. With more molecules or atoms in the upper than in the lower state, there can be amplification. Although doing this was clearly not easy, I recognized practical methods of doing it.

The simplest and best way I could be sure of having more molecules in the upper than in the lower state was by separating them in a molecular beam, a technique worked on at Columbia by Professors Rabi, Kusch, and others. I decided to first try using ammonia to amplify microwaves, since I had a lot of microwave equipment and had carefully studied ammonia at these frequencies. A student, Jim Gordon, agreed to do his thesis attempting to amplify with ammonia. And, with a post doc Herb Zeiger, he made it work after a little more than two years of preparation. While this was underway, it was not of much interest to other scientists. And Professors Rabi and Kusch (the present and past heads of our physics department), after we had worked on it for almost two years, came into my lab and said "Charlie, you really must stop! Thats not going to work. We know it wont and you do too. Youre wasting the departments money". But Jim Gordon and I stuck to it, and soon it worked! We named it the maser for microwave amplification of radiation by stimulated emission of radiation, and on publication of the result providing a very pure frequency oscillator and the worlds most sensitive amplification of microwaves this became a hot field, with much excitement and competition.

Somewhat before the maser was invented, Prof. Willis Lamb of Columbias physics department had measured the fine structure of hydrogen using an atomic beam of hydrogen and microwaves of $21 \mathrm{~cm}$. wavelengths. It importantly showed a deviation from simple atomic theory. And for this, he was later awarded the Nobel Prize. I decided to look for hydrogen atoms in space, using a maser amplifier to sensitively detect this wavelength, and persuaded my graduate student Arno Penzias to look for hydrogen in interstellar space as his thesis. He didnt succeed, but did finish a Ph.D. degree, and took a job at Bell Telephone Laboratories where he decided to continue to look for hydrogen. Robert Wilson worked with him. They didnt find hydrogen, but the sensitive maser amplifier 
used allowed them to note a weak continuum microwave radiation present in all directions across the sky a surprising puzzle which was soon understood to be a product of the big bang evidence for the origin of the universe! That of course also won a Nobel Prize what could be more exciting than discovery of evidence for the universes beginning?

At about that time I had also, with Arthur Schawlow, shown how the maser idea could be extended to the infrared and optical region. Getting into the infrared region had been my primary goal when the idea of using stimulated emission came to me. And soon the laser came along, with the new name meaning light amplification by stimulated emission of radiation. Lasers now produce wavelengths from the far infrared, just short of microwaves (where they are called masers), on into the far ultraviolet and $x$-ray regions. Masers and lasers represented such a hot field with so many participants that I felt I wasnt needed any more, and decided to go rather fully into astrophysics, where I thought I saw some new and neglected possibilities using infrared and microwaves, at which wavelengths not much astronomy was then being done. I decided for one thing to look for molecules in space using microwaves, and picked out ammonia as my first target. I had moved to the University of California where George Field, an outstanding astrophysics theorist, was then chairman of the department. He told me I was wasting my time looking for molecules in interstellar space. They could not be there, as he said he could prove theoretically. Nevertheless, I looked for them, typical of my willingness to differ with experts. And sure enough, there was ammonia. By now, microwave spectra from many, many different molecules in space have been detected, and study of clouds of molecules has become a rich field. Also radiation from many masers produced in space has shown up as well as from a few lasers. Use of microwaves is now continuously yielding much interesting astrophysics.

High resolution spatial interferometry for studying stars was introduced by Michelson back in the 1920s. Such work at optical or infrared wavelengths was highly regarded, but was limited in use and resolution because two separate telescopes could not do such interferometry. This was because they could not be sufficiently stabilized in their separation. Now, however, lasers are able to stabilize the distance between telescopes and the field of interferometry for astronomy is growing rapidly at all wavelengths.

I myself have been doing stellar interferometry in the mid infrared region, using lasers both for control of distances and for heterodyne detection of the radiation. Interferometry for astronomy extends from the visible, through the infrared, to sub millimeter and microwavelengths, and results are often closely related over this broad wavelength range.

Many people have contributed to scientific and technical developments in the visible and infrared regions, particularly with lasers. These developments have been important in extending very active research and various types of ideas and techniques more-or-less continuously throughout the optical, infrared, and far infrared regions. With the above science mentioned, plus many other developments, the field of electromagnetic waves has been developing rapidly, with techniques and ideas spreading, unifying the science and use of electromagnetic waves at all wavelengths, from $x$-rays through the radio region. There is now no sharp break in ideas or techniques at any wavelength. 